\title{
Synthesis of Spirocyclopente-Dione Anthracene Adduct, Precursor of the Cyclopentenone Prostagladins Via Ring-Closing Metathesis Reaction
}

\author{
GEDSIRIN EKSINITKUN ${ }^{\mathrm{a}, \mathrm{b}}$, STEPHEN G. PYNE ${ }^{\mathrm{b}}$, \\ WAYA PHUTDHAWONG ${ }^{\mathrm{a}}$ and WEERACHAI PHUTDHAWONG ${ }^{\mathrm{c} *}$
}

${ }^{\mathrm{a}}$ Department of Chemistry, Faculty of Science, Silpakorn University, Nakhon Pathom-73000, Thailand

${ }^{\mathrm{b}}$ School of Chemistry, University of Wollongong, Wollongong, N.S.W-2522, Australia

${ }^{c}$ Department of Chemistry, Faculty of Liberal Arts and Science, Kasetsart University, Kampeang Sean Campus, Nakhon Pathom-73140, Thailand

faaswcp@ku.ac.th

Received 24 April 2013 / Accepted 7 May 2013

\begin{abstract}
A synthesis of the spirocyclopente-dione anthracene adduct, a precursor of the cyclopentenone prostaglandins has been reported. The synthesis involved a Diels-Alder reaction of anthracene and dimethyl fumarate to afford $\mathbf{3}$ followed by reduction, oxidation and esterification reactions to provide methyl ester anthracene adduct $\mathbf{8}$, which further converted to the the allylic alcohol (12). Then, Ring-Closing Metathesis (RCM) reaction afforded the cyclopentenol anthracene adduct, which after oxidation provided the spirocyclopente-dione anthracene adduct in good yields.
\end{abstract}

Keywords: Cross-conjugated, Dienone, Ring closing metathesis, Cyclopentenones

\section{Introduction}

Functionalized cyclopentenones are considered important precursors in the synthesis of a large number of bioactive natural products such as conjugated dienone prostanoids, clavulone I, clavulone $\mathrm{II}^{1-2}$ and claviridic acids $\mathrm{A}-\mathrm{E}^{3}$, all exhibiting strong cytotoxicity and anticancer activity. Several synthetic approaches have been proposed to prepare this class of compounds including the Nazarov cyclisation ${ }^{4},[3+2]$ annulations, metal-catalysed cyclisations $^{6}$ and Diels-Alder/retro Diels-Alder reactions using anthracene ${ }^{7}$. In our previous research of stereoselective synthesized cyclopentenone ${ }^{8}$, we found the use of sterically crowded anthracene moiety leads to stereocontrol and asymmetric transformations and subsequent retro-Diels-Alder reaction affords alkylidene cyclopentenone A (Figure 1), a precursor of the antitumor alkylidene cyclopentenone prostaglandins (PGs) such as clavulone $\mathrm{II}^{2}$ and 4-deacetoxyl-12-O-deacetylclavulone $\mathrm{III}^{9}$. However, the cyclization process using $\beta$-allyl ester with an excess of LDA provided cyclopentenone in moderate yields together with the formation of the enaminone ${ }^{10}$. As an extension, we here report a 
novel building block for the synthesis of cyclopentenone prostaglandins, in the particular $\mathrm{PGA}_{1}$ analog clavulones. The synthesis of this key compound, spirocyclopente-dione anthracene adduct, could be envisioned from the cyclopentenol readily obtained from the 1,6-diene using a ring-closing metathesis reaction.

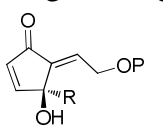

A

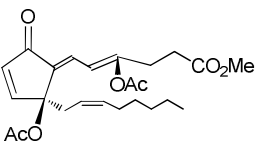

Clavulone II

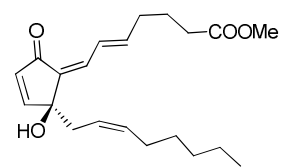

4-deacetoxyl-12-O-deacetylclavulone III

Figure 1

\section{Experimental}

Melting points were determined on a Stuart Scientific SMP 2 melting point apparatus and are uncorrected. Infrared spectra were recorded as $\mathrm{CH}_{2} \mathrm{Cl}_{2}$-films with a Perkin Elmer Spectrum GX FT-IR spectrophotometer. ${ }^{1} \mathrm{H}$ - and ${ }^{13} \mathrm{C}-\mathrm{NMR}$ spectra were recorded in (D) chloroform solutions at $300 \mathrm{MHz}$ for ${ }^{1} \mathrm{H}$ and $75 \mathrm{MHz}$ for ${ }^{13} \mathrm{C}$ with a Bruker AVANCE 300 spectrometer. Tetramethylsilane was used as the internal standard. Mass spectra were recorded on a Polaris Q or Hewlett Packard 5973 mass spectrometer.

\section{9,10-Dihydro-9,10-ethanoanthracene-11,12-dimethyl ester (3)}

A mixture of anthracene $(2.00 \mathrm{~g}, 11.22 \mathrm{mmol})$, dimethyl fumarate $(2.05 \mathrm{~g}, 14.23 \mathrm{mmol})$ and xylene $(15 \mathrm{~mL})$ in a pressured tube with boiling chips was heated at $120{ }^{\circ} \mathrm{C}$ for $48 \mathrm{~h}$. The reaction mixture was cooled to room temperature and the xylene was then removed under vacuo. The crude product was purified using column chromatography (silica gel, 30:1 hexane/EtOAc) to afford the adduct $3(2.82 \mathrm{~g}, 78 \%)$ as a white solid, m.p. $103-105^{\circ} \mathrm{C}$ (lit. ${ }^{11}$ 107-108 $\left.{ }^{\circ} \mathrm{C}\right)$; IR $\left(\mathrm{CH}_{2} \mathrm{Cl}_{2}\right) \nu_{\max }: 1732,1459,1435,1221,1198,1018,760 \mathrm{~cm}^{-1} ;{ }^{1} \mathrm{H}$ NMR $\delta$ 7.28-7.31 (m, 2H, ArH), 7.22-7.25 (m, 2H), 7.07-7.14 (m, 4H), $4.73(\mathrm{~s}, 2 \mathrm{H}), 3.62(\mathrm{~s}, 6 \mathrm{H})$, $3.42(\mathrm{~s}, 2 \mathrm{H}) ;{ }^{13} \mathrm{C}$ NMR $\delta 172.8,142.0,140.3,126.4,126.3,124.6,123.8,52.2,47.8,46.7$.

\section{9,10-Dihydro-9,10-ethanoanthracene-11,12-dimethyl alcohol (4)}

To a solution of $3(2.75 \mathrm{~g}, 8.54 \mathrm{mmol})$ in THF $(60 \mathrm{~mL})$ at $0{ }^{\circ} \mathrm{C}$ lithium aluminium hydride $(1.94 \mathrm{~g}, 51.2 \mathrm{mmol})$ was slowly added. The mixture was stirred at $0{ }^{\circ} \mathrm{C}$ under argon atmosphere for $30 \mathrm{~min}$. The reaction mixture was quenched with sat. $\mathrm{NaHCO}_{3}$ solution and extracted with $\mathrm{Et}_{2} \mathrm{O}(3 \times 30 \mathrm{~mL})$. The combined organic phase was dried $\left(\mathrm{Na}_{2} \mathrm{SO}_{4}\right)$, filtered and concentrated under reduced pressure to give the diol $4(2.15 \mathrm{~g}, 98 \%)$ as a white solid; m.p. 196-198 ${ }^{\circ} \mathrm{C}$; IR $\left(\mathrm{CH}_{2} \mathrm{Cl}_{2}\right) v_{\max }: 3442,3054,2987,1422,1022 \mathrm{~cm}^{-1} ;{ }^{1} \mathrm{H}$ NMR $\delta 7.38-$ 7.35 (m, 4H), 7.18-7.15 (m, 4H), 4.44 (s, 2H), 3.22-3.17 (m, 2H), 2.89-2.81(m, 2H), 1.381.33 (m, 2H); ${ }^{13} \mathrm{C}$ NMR $\delta 144.5,141.7,126.1,125.8,123.4,64.5,45.9,45.3$; HRESI-MS $m / z$ cald for $[\mathrm{M}+\mathrm{Na}]^{+} \mathrm{C}_{18} \mathrm{H}_{18} \mathrm{NaO}_{2}: 289.1193$, found: 289.1182 .

\section{9,10-Dihydro-9,10-ethanoanthracene-11-acetoxy-12-methanol (5)}

To a solution of the diol $4(5.80 \mathrm{~g}, 21.8 \mathrm{mmol})$ in DMF $(8 \mathrm{~mL})$ at room temperature pyridine (2.11 $\mathrm{mL}, 26.2 \mathrm{mmol})$ was added followed by acetic anhydride $(2.06 \mathrm{~mL}, 21.8 \mathrm{mmol})$. The mixture was stirred at room temperature for $30 \mathrm{~min}$. The reaction mixture was quenched with water and extracted with $\mathrm{CH}_{2} \mathrm{Cl}_{2}(2 \times 20 \mathrm{~mL})$. The combined extracts were dried $\left(\mathrm{Na}_{2} \mathrm{SO}_{4}\right)$, filtered and concentrated under reduced pressure. The crude product was purified by flash column chromatography (silica gel, 3:1 hexane/ EtOAc) to give the mono-acetate $5(3.48 \mathrm{~g}, 52 \%)$ as a white solid; m.p. 89-91 ${ }^{\circ} \mathrm{C}$; IR $\left(\mathrm{CH}_{2} \mathrm{Cl}_{2}\right) v_{\max }: 3451,3054,2987,1736,1422,1025 \mathrm{~cm}^{-1} ;{ }^{1} \mathrm{H}$ NMR $\delta 7.27-$ $7.23(\mathrm{~m}, 4 \mathrm{H}), 7.11-7.07(\mathrm{~m}, 4 \mathrm{H}), 4.32(\mathrm{~d}, J=1.8 \mathrm{~Hz}, 1 \mathrm{H}), 4.21(\mathrm{~d}, J=1.8 \mathrm{~Hz}, 1 \mathrm{H}), 3.81(\mathrm{dd}, J=10.6$, 
$5.8 \mathrm{~Hz}, 1 \mathrm{H}), 3.51(\mathrm{dd}, J=10.4,8.7 \mathrm{~Hz}, 1 \mathrm{H}), 3.27(\mathrm{dd}, J=10.6,5.8 \mathrm{~Hz}, 1 \mathrm{H}), 3.06(\mathrm{dd}, J=10.4$, $8.7 \mathrm{~Hz}, 1 \mathrm{H}), 2.48$ (br. s, $1 \mathrm{H}), 2.03$ (s, 3H), 1.66-1.52 (m, 2H); ${ }^{13} \mathrm{C}$ NMR $\delta 171.2,143.5,143.1$, $140.7,140.4,126.3,126.2,125.9,125.8,125.5,125.4,123.5 * 2,67.1,65.5,45.9,45.7,45.5$, 42.2, 21.0; HRESI-MS $m / z$ cald for [M+Na] ${ }^{+} \mathrm{C}_{20} \mathrm{H}_{20} \mathrm{NaO}_{3}: 331.1310$, found: 331.1306 .

\section{9,10-Dihydro-9,10-ethanoanthracene-11-acetoxy-12-acetic acid (6)}

A solution of alcohol $5(0.22 \mathrm{~g}, 0.71 \mathrm{mmol})$ in acetone $(6 \mathrm{~mL})$ was treated with Jones reagent ${ }^{18}$ $(4 \mathrm{~mL})$ at $0{ }^{\circ} \mathrm{C}$ until TLC analysis showed the reaction was complete $(c a .1 \mathrm{~h})$. Isopropanol $(0.6 \mathrm{~mL})$ was added slowly drop wise to destroy excess reagent and the mixture was stirred for another 5-10 min until the colour of the solution changed from red to green. $\mathrm{CH}_{2} \mathrm{Cl}_{2}(20 \mathrm{~mL})$ and water $(20 \mathrm{~mL})$ were added. The aqueous phase was extracted with $\mathrm{CH}_{2} \mathrm{Cl}_{2}(10 \mathrm{~mL} \times 3)$. The combined organic extracts were washed with water $(40 \mathrm{~mL})$ and brine $(40 \mathrm{~mL})$ and then died $\left(\mathrm{Na}_{2} \mathrm{SO}_{4}\right)$, filtered and evaporated in vacuo to give compound $6(0.21 \mathrm{~g}, 91 \%)$ as a yellow oil; IR $\left(\mathrm{CH}_{2} \mathrm{Cl}_{2}\right) v_{\max }: 3436,2987,1737,1708,1422,1036 \mathrm{~cm}^{-1} ;{ }^{1} \mathrm{H}$ NMR $\delta$ 7.35-7.28 (m, 4H), 7.18-7.12 $(\mathrm{m}, 4 \mathrm{H}), 4.68(\mathrm{~d}, J=2.1 \mathrm{~Hz}, 1 \mathrm{H}), 4.33(\mathrm{~d}, J=2.1 \mathrm{~Hz}, 1 \mathrm{H}), 3.89-3.84(\mathrm{~m}, 1 \mathrm{H}), 3.77-3.70(\mathrm{~m}$, 1H), 2.73-2.65 (m, $1 \mathrm{H}), 2.42(\mathrm{dd}, J=5.6,2.3 \mathrm{~Hz}, 1 \mathrm{H}), 2.60(\mathrm{~s}, 3 \mathrm{H}) ;{ }^{13} \mathrm{C}$ NMR $\delta 178.1,171.0$, $143.3,141.9,140.2,139.9,126.4 * 3,126.1,125.5,125.3,123.6,123.5,66.7,48.2,46.3$, 45.9, 41.7, 20.9; HRESI-MS $m / z$ cald for $[\mathrm{M}+\mathrm{Na}]^{+} \mathrm{C}_{20} \mathrm{H}_{18} \mathrm{NaO}_{4}: 345.1103$, found: 345.1089 .

\section{9,10-Dihydro-9,10-ethanoanthracene-11-methanol-12-methyl ester (7)}

To a solution of 6 ( $2.34 \mathrm{~g}, 7.27 \mathrm{mmol})$ in methanol $(14 \mathrm{~mL})$ was added drop wise conc. $\mathrm{H}_{2} \mathrm{SO}_{4}$ $(1 \mathrm{~mL})$. The mixture was stirred at room temperature for $12 \mathrm{~h}$. The reaction mixture was quenched with water and extracted with $\mathrm{CH}_{2} \mathrm{Cl}_{2}(3 \times 20 \mathrm{~mL})$. The combined extracts were dried $\left(\mathrm{Na}_{2} \mathrm{SO}_{4}\right)$, filtered and concentrated to give $7(1.82 \mathrm{~g}, 85 \%)$ as a yellow oil; $\mathrm{IR}\left(\mathrm{CH}_{2} \mathrm{Cl}_{2}\right) v_{\max }$ : $3443,2987,1733,1422,1023 \mathrm{~cm}^{-1}$; ${ }^{1} \mathrm{H}$ NMR $\delta 9.20$ (br. s, 1H), 7.32-7.23 (m, 4H), 7.11-7.05 $(\mathrm{m}, 4 \mathrm{H}), 4.62(\mathrm{~d}, J=2.1 \mathrm{~Hz}, 1 \mathrm{H}), 4.31(\mathrm{~d}, J=2.1 \mathrm{~Hz}, 1 \mathrm{H}), 3.27(\mathrm{~s}, 3 \mathrm{H}) 3.09-2.96(\mathrm{~m}, 2 \mathrm{H})$, 2.58-2.50 (m, 1H), $2.22(\mathrm{dd}, J=5.7,2.2 \mathrm{~Hz}, 1 \mathrm{H}) ;{ }^{13} \mathrm{C}$ NMR $\delta$ 177.2, 143.6, 142.4, 140.7, $140.2,126.3,126.28,126.25,126.0,125.6,125.5,123.5,123.4,75.8,58.9,48.7,46.1,45.6$, 42.7; HRESI-MS $m / z$ cald for $[\mathrm{M}+\mathrm{Na}]^{+} \mathrm{C}_{19} \mathrm{H}_{18} \mathrm{NaO}_{3}: 317.1154$, found: 317.1148 .

\section{1-(tert-Butyl-dimethyl-silanyloxy)methyl-9,10-dihydro-9,10-ethanoanthracene-12-} methyl ester (8)

To a solution of alcohol $7(1.50 \mathrm{~g}, 5.09 \mathrm{mmol})$ in dry $\mathrm{CH}_{2} \mathrm{Cl}_{2}(43 \mathrm{~mL})$ under an argon atmosphere imidazole $(0.66 \mathrm{~g}, 10.2 \mathrm{mmol})$ was added followed by TBDMSCl $(0.88 \mathrm{~g}, 5.61 \mathrm{mmol})$. The mixture was stirred at room temperature for $15 \mathrm{~h}$. The reaction mixture was quenched with sat. $\mathrm{NaHCO}_{3}$ solution and extracted with $\mathrm{CH}_{2} \mathrm{Cl}_{2}(2 \times 30 \mathrm{~mL})$. The combined extracts were dried $\left(\mathrm{Na}_{2} \mathrm{SO}_{4}\right)$, filtered and concentrated. Purification by flash column chromatography (silica gel, 30:1 hexane/ EtOAc) gave silyl ether 8 (1.68 g, 81\%) as a white solid, m.p. 81.7-84.8 ${ }^{\circ} \mathrm{C}$; IR $\left(\mathrm{CH}_{2} \mathrm{Cl}_{2}\right) v_{\max }: 3445,2953,1737,1470,1253,1213,1094,837,746 \mathrm{~cm}^{-1} ;{ }^{1} \mathrm{H}$ NMR $\delta 7.21-$ $7.13(\mathrm{~m}, 4 \mathrm{H}), 7.03-6.98(\mathrm{~m}, 4 \mathrm{H}), 4.48(\mathrm{~d}, J=2.3 \mathrm{~Hz}, 1 \mathrm{H}), 4.30(\mathrm{~d}, J=2.3 \mathrm{~Hz}, 1 \mathrm{H}), 3.20$ (s, $\left.3 \mathrm{H}, \mathrm{OCH}_{3}\right), 3.12-3.07(\mathrm{~m}, 1 \mathrm{H}), 2.64(\mathrm{~d}, J=9.1 \mathrm{~Hz}, 1 \mathrm{H}), 2.58-2.52(\mathrm{~m}, 1 \mathrm{H}), 2.06-2.04(\mathrm{~m}$, $1 \mathrm{H}), 0.80(\mathrm{~s}, 9 \mathrm{H}), 0.08(\mathrm{~s}, 3 \mathrm{H}), 0.04(\mathrm{~s}, 3 \mathrm{H}) ;{ }^{13} \mathrm{C}$ NMR $\delta 173.5,144.1,142.6,141.1,140.8$, $126.4,126.2 * 2,125.9,125.8,125.1,123.6,123.5,65.7,61.9,48.0,46.9,45.7,45.6,26.1$; HRESI-MS $m / z$ cald for $[\mathrm{M}+\mathrm{Na}]^{+} \mathrm{C}_{25} \mathrm{H}_{32} \mathrm{NaO}_{3} \mathrm{Si}$ : 431.2018, found: 431.2049.

\section{1-(tert-Butyl-dimethyl-silanyloxy)methanol-9,10-dihydro-9,10-ethanoanthracene- 12,12-dimethylester (9)}

To a solution of $2 \mathrm{M}$ lithium diisopropylamide $(0.74 \mathrm{~mL}, 1.47 \mathrm{mmol})$ in dry THF $(2.5 \mathrm{~mL})$ at $-78{ }^{\circ} \mathrm{C}$ under argon atmosphere a solution of ester $8(0.50 \mathrm{~g}, 1.22 \mathrm{mmol})$ was added in dry 
THF (5 mL) and the mixture was allowed to warm to $0{ }^{\circ} \mathrm{C}$ for $2 \mathrm{~h}$. Methyl cyanoformate $(0.12 \mathrm{~mL}, 1.47 \mathrm{mmol})$ was added at $-78{ }^{\circ} \mathrm{C}$ and the mixture stirred at $0{ }^{\circ} \mathrm{C}$ for $2 \mathrm{~h}$. The resulting mixture was quenched with an aqueous saturated $\mathrm{NaHCO}_{3}(20 \mathrm{~mL})$ and extracted with $\mathrm{CH}_{2} \mathrm{Cl}_{2}(2 \times 15 \mathrm{~mL})$ which was washed with water $(20 \mathrm{~mL})$ and saturated $\mathrm{NaCl}(20 \mathrm{~mL})$. The combined extracts were dried $\left(\mathrm{Na}_{2} \mathrm{SO}_{4}\right)$, filtered and concentrated in vacuo. Purification of the residue by flash column chromatography (silica gel, 25:1 hexane/ EtOAc) gave 9 $(0.41 \mathrm{~g}, 81 \%)$ as a white solid, m.p. $129-131^{\circ} \mathrm{C}$; IR $\left(\mathrm{CH}_{2} \mathrm{Cl}_{2}\right) \mathrm{v}_{\max }: 2952,1741,1459,1220$, 1095, $837 \mathrm{~cm}^{-1}$; ${ }^{1} \mathrm{H}$ NMR $\delta 7.44(\mathrm{~d}, J=7.3 \mathrm{~Hz}, 1 \mathrm{H}), 7.30(\mathrm{~d}, J=7.3 \mathrm{~Hz}, 1 \mathrm{H}), 7.23(\mathrm{~d}, J=7.1$ $\mathrm{Hz}, 1 \mathrm{H}), 7.14(\mathrm{~d}, J=7.1,5.5 \mathrm{~Hz}, 2 \mathrm{H}), 7.10(\mathrm{t}, J=7.3 \mathrm{~Hz}, 2 \mathrm{H}), 7.04(\mathrm{dd}, J=7.5,7.3 \mathrm{~Hz}$, $1 \mathrm{H}), 4.8(\mathrm{~s}, 1 \mathrm{H}), 4.58(\mathrm{~d}, J=2.4 \mathrm{~Hz}, 1 \mathrm{H}), 3.57(\mathrm{~s}, 3 \mathrm{H}), 3.55(\mathrm{~s}, 3 \mathrm{H}), 3.54-3.51(\mathrm{~m}, 1 \mathrm{H})$, 3.30-3.22 (m, 1H), $2.67(\mathrm{dd}, J=10.8,9.5 \mathrm{~Hz}, 1 \mathrm{H}), 0.93(\mathrm{~s}, 9 \mathrm{H}), 0.00(\mathrm{~s}, 6 \mathrm{H}),{ }^{13} \mathrm{C}$ NMR $\delta$ $170.7,170.1,143.9,141.6,140.5,140.1,127.0,126.9,126.1,126.0,125.9,125.3,124.5$, $124.4,62.4,61.9,53.1,52.3,51.0,47.1,45.6,26.2,-5.16,-5.2$. HRESI-MS $m / z$ cald for [M-H] ${ }^{-} \mathrm{C}_{27} \mathrm{H}_{33} \mathrm{O}_{5} \mathrm{Si}: 465.2097$, found: 465.2118 .

\section{1-(tert-Butyl-dimethyl-silanyloxy)methanol-9,10-dihydro-9,10-ethanoanthracene- 12,12-dimethanol (10)}

To adduct $9(0.16 \mathrm{~g}, 0.33 \mathrm{mmol})$ in $\mathrm{Et}_{2} \mathrm{O}(2 \mathrm{~mL})$ at $0{ }^{\circ} \mathrm{C}$ lithium aluminium hydride (53 mg, $1.4 \mathrm{mmol}$ ) was slowly added. The mixture was stirred at $0{ }^{\circ} \mathrm{C}$ under argon atmosphere for $30 \mathrm{~min}$. The reaction was quenched with saturated $\mathrm{NaHCO}_{3}$ solution and extracted with $\mathrm{Et}_{2} \mathrm{O}(3 \times 30 \mathrm{~mL})$. The combined extracts were dried $\left(\mathrm{Na}_{2} \mathrm{SO}_{4}\right)$, filtered and concentrated under reduced pressure to give a diol $10(74 \mathrm{mg}, 61 \%)$ as a white solid, m.p. 204-206 ${ }^{\circ} \mathrm{C}$; IR $\left(\mathrm{CH}_{2} \mathrm{Cl}_{2}\right) v_{\max }: 3262,2929,1466,1253,1059,835 \mathrm{~cm}^{-1} ;{ }^{1} \mathrm{H}$ NMR $\delta 7.37-$ $7.34(\mathrm{~m}, 1 \mathrm{H}), 7.28-7.19(\mathrm{~m}, 3 \mathrm{H}), 7.13-7.08(\mathrm{~m}, 4 \mathrm{H}), 4.35(\mathrm{~s}, 1 \mathrm{H}), 4.06(\mathrm{~d}, J=1.78 \mathrm{~Hz}, 1 \mathrm{H})$, $3.63(\mathrm{dd}, J=10.8,5.2 \mathrm{~Hz}, 2 \mathrm{H}), 3.47(\mathrm{dd}, J=10.6,1.6 \mathrm{~Hz}, 1 \mathrm{H}), 3.35-3.32(\mathrm{~m}, 3 \mathrm{H}), 2.96(\mathrm{dd}$, $J=10.7,10.3 \mathrm{~Hz}, 1 \mathrm{H}), 2.65$ (br.s, 1H), 1.52 (qd, $J=5.1,1.9 \mathrm{~Hz}, 1 \mathrm{H}), 0.86(\mathrm{~s}, 9 \mathrm{H}), 0.066$ (s, 3H), 0.044 (s, 3H). ${ }^{13} \mathrm{C}$ NMR $\delta 143.6,141.8,141.4,140.9,126.3,126.2 * 2,126.0,125.8$, 125.3, 124.9, 123.2, 68.2, 65.3, 65.2, 49.4, 48.5, 48.1, 47.9, 26.0, -5.13, -5.29. HRCI-MS m/z cald for $[\mathrm{M}+\mathrm{H}]^{+} \mathrm{C}_{25} \mathrm{H}_{35} \mathrm{O}_{3} \mathrm{Si}: 411.2355$, found:411.2361.

\section{1-(tert-Butyl-dimethyl-silanyloxy)methanol-9,10-dihydro-9,10-ethanoanthracene- 12,12-dicarbaldehyde (11)}

To a solution of alcohol $10(0.10 \mathrm{~g}, 0.24 \mathrm{mmol})$ in dry $\mathrm{CH}_{2} \mathrm{Cl}_{2}(10 \mathrm{~mL})$ under an argon atmosphere Dess-Martin periodinane $(0.36 \mathrm{~g}, 0.85 \mathrm{mmol})$ was added. The mixture was stirred at room temperaaature for $2 \mathrm{~h}$. The reaction was quenched with sat. $\mathrm{NaHCO}_{3}$ and $\mathrm{NaS}_{2} \mathrm{O}_{3} .5 \mathrm{H}_{2} \mathrm{O}$ solution $(10 \mathrm{~mL})$ and extracted with $\mathrm{CH}_{2} \mathrm{Cl}_{2}(2 \times 20 \mathrm{~mL})$. The combined extracts were dried $\left(\mathrm{Na}_{2} \mathrm{SO}_{4}\right)$, filtered and concentrated to give dialdehyde $\mathbf{1 1}(94 \mathrm{mg}, 95 \%)$ as a yellow oil; ${ }^{1} \mathrm{H}$ NMR $\delta 9.66(\mathrm{~s}, 1 \mathrm{H}), 9.10(\mathrm{~s}, 1 \mathrm{H}), 7.39(\mathrm{~d}, J=6.3 \mathrm{~Hz}, 2 \mathrm{H}), 7.35(\mathrm{~d}, J=7.3 \mathrm{~Hz}, 1 \mathrm{H})$, $7.31(\mathrm{~d}, J=7.3 \mathrm{~Hz}, 1 \mathrm{H}), 7.23(\mathrm{t}, J=6.3 \mathrm{~Hz}, 2 \mathrm{H}), 7.2-7.12(\mathrm{~m}, 2 \mathrm{H}), 4.82(\mathrm{~s}, 1 \mathrm{H}), 4.38(\mathrm{~s}, 1 \mathrm{H})$, $3.76(\mathrm{dd}, J=9.8,7.3 \mathrm{~Hz}, 1 \mathrm{H}), 3.48(\mathrm{t}, J=9.8 \mathrm{~Hz}, 1 \mathrm{H}),, 2.99(\mathrm{dd}, J=9.3,7.3 \mathrm{~Hz}, 1 \mathrm{H}), 0.85$ (s, 9H), 0.05 (s, 3H), 0.01(s, 3H). ${ }^{13} \mathrm{C}$ NMR $\delta$ 201.1, 198.4, 143.8, 142.2, 138.4, 138.37, 127.0, $126.9,126.5,126.3,125.3,125.1,123.6,66.5,62.5,49.2,46.8,46.2,25.8,-5.5,-5.8$.

\section{1-((tert-Butyl-dimethyl-silanyloxy)methanol-9,10-dihydro-9,10-ethanoanthracene- 12,12-diyl)bis(prop-2-en-1-ol) (12)}

Vinyl magnesium bromide $(1.9 \mathrm{~mL}, 1.91 \mathrm{mmol})$ was added drop wise to the solution of the aldehyde $11(0.15 \mathrm{~g}, 0.38 \mathrm{mmol})$ in $\operatorname{dry~}^{2} \mathrm{Et}_{2} \mathrm{O}(5 \mathrm{~mL})$ at $-78{ }^{\circ} \mathrm{C}$ under argon atmosphere. The mixture was stirred at $-78^{\circ} \mathrm{C}$ for $40 \mathrm{~min}$. The reaction was quenched with sat. $\mathrm{NH}_{4} \mathrm{Cl}$ 
at $-78{ }^{\circ} \mathrm{C}$ and allowed to warm to room temperature. The mixture was extracted with $\mathrm{Et}_{2} \mathrm{O}(2 \times 20 \mathrm{~mL})$. The combined organic layers were washed with brine, dried over anhyd. $\mathrm{Na}_{2} \mathrm{SO}_{4}$, filtered and concentrated. Purification of the residue by flash column chromatography (silica gel, 20:1 hexane/ EtOAc) gave $\mathbf{1 2}(16 \mathrm{mg}$ g, 8.9\%) as a yellow oil; IR $\left(\mathrm{CH}_{2} \mathrm{Cl}_{2}\right) v_{\max }: 3350,2929,1469,1254,1086,836 \mathrm{~cm}^{-1} ;{ }^{1} \mathrm{H}$ NMR $\delta{ }^{1} \mathrm{H} ; 7.36-$ $7.34(\mathrm{~m}, 1 \mathrm{H}), 7.25-7.22(\mathrm{~m}, 2 \mathrm{H}), 7.10-7.07(\mathrm{~m}, 5 \mathrm{H}), 6.33-6.26(\mathrm{~m}, 2 \mathrm{H}), 6.24-6.18$ $(\mathrm{m}, 2 \mathrm{H}), 5.22(\mathrm{dd}, J=17.1,10.4 \mathrm{~Hz}, 2 \mathrm{H}), 5.06(\mathrm{dd}, J=17.1,10.4 \mathrm{~Hz}, 2 \mathrm{H}), 4.71(\mathrm{~s}, 1 \mathrm{H})$, $4.29(\mathrm{~d}, J=1.5 \mathrm{~Hz}, 1 \mathrm{H}), 3.79(\mathrm{~d}, J=6.5 \mathrm{~Hz}, 1 \mathrm{H}), 3.69-3.65(\mathrm{dd}, J=10.7,7.6 \mathrm{~Hz}, 1 \mathrm{H})$, $3.50(\mathrm{dd}, J=10.8,6.9 \mathrm{~Hz}, 1 \mathrm{H}), 3.32(\mathrm{~d}, J=5.0 \mathrm{~Hz}, 1 \mathrm{H}), 1.97(\mathrm{t}, J=7.2 \mathrm{~Hz}, 1 \mathrm{H}), 0.92$ $(\mathrm{s}, 9 \mathrm{H}), 0.096(\mathrm{~s}, 3 \mathrm{H}), 0.065(\mathrm{~s}, 3 \mathrm{H}) .{ }^{13} \mathrm{C}$ NMR $\delta 143.9,142.1,141.9,141.8,139.5$, $139.4,126.3,126.1,126.0,125.9,125.84,125.82,125.1,123.4,117.1,114.9,80.5$, $76.9,65.2,53.9,49.3,48.3,48.26,26.1,-5.1,-5.2$. HRESI-MS $\mathrm{m} / \mathrm{z}$ cald for [M] $\mathrm{C}_{29} \mathrm{H}_{38} \mathrm{O}_{3} \mathrm{Si}: 462.2590$, found: 462.2613 .

\section{1-((tert-Butyl-dimethyl-silanyloxy)methanol-9,10-dihydrospiro-[9,10-} ethanoanthracene -12,1'-cyclopen[3]ene]-2',5'-diol (13)

To a solution of compound $12(0.11 \mathrm{~g}, 0.25 \mathrm{mmol})$ Grubb I catalyst $(10 \mathrm{mg}, 0.012 \mathrm{mmol})$ in $\mathrm{CH}_{2} \mathrm{Cl}_{2}(4.3 \mathrm{~mL})$ was added. The reaction was heated at $40{ }^{\circ} \mathrm{C}$ under argon atmosphere for $15 \mathrm{~h}$ and then concentrated under reduced pressure. The crude product was purified by Flash column chromatography (silica gel, 6:1 hexane/ EtOAc) to give compound $\mathbf{1 3}$ (56 mg, 53\%) as a yellow oil; ${ }^{1} \mathrm{H}$ NMR $\delta$ 7.39-7.37 $(\mathrm{m}, 3 \mathrm{H}), 7.31(\mathrm{~d}, J=6 \mathrm{~Hz}, 1 \mathrm{H}), 7.19-7.11(\mathrm{~m}, 4 \mathrm{H}), 6.01$ $(\mathrm{d}, J=6 \mathrm{~Hz}, 1 \mathrm{H}), 5.91-5.90(\mathrm{~m}, 1 \mathrm{H}), 5.08(\mathrm{~s}, 1 \mathrm{H}), 4.59(\mathrm{~s}, 1 \mathrm{H}), 4.53(\mathrm{~s}, 1 \mathrm{H}), 3.83(\mathrm{~s}, 1 \mathrm{H}), 3.54$ $(\mathrm{dd}, J=10.7,1.2 \mathrm{~Hz}, 1 \mathrm{H}), 3.99(\mathrm{t}, J=10.7 \mathrm{~Hz}, 1 \mathrm{H}), 1.77(\mathrm{~d}, J=9.8 \mathrm{~Hz}, 1 \mathrm{H}), 0.93(\mathrm{~s}, 9 \mathrm{H})$, 0.057 (s, 3H), 0.00 (s, 3H). ${ }^{13} \mathrm{C}$ NMR $\delta 144.9,143.4,142.2,141.7,141.3,131.6,126.6,126.59$, $126.4,126.3,125.9,124.4,123.5,123.45,85.2,83.1,64.9,58.8,55.6,50.8,46.8,26.2,-4.9$, 5.0. HRESI-MS $m / z$ cald for $[\mathrm{M}-\mathrm{H}]]^{-} \mathrm{C}_{27} \mathrm{H}_{33} \mathrm{O}_{3} \mathrm{Si}: 433.2199$, found: 433.2174 .

\section{1-((tert-Butyl-dimethyl-silanyloxy)methanol-9,10-dihydrospiro-[9,10-} ethanoanthracene -12,1'-cyclopen[3]ene]-2',5'-dione (14)

To a solution of alcohol $13(15 \mathrm{mg}, 0.035 \mathrm{mmol})$ in dry $\mathrm{CH}_{2} \mathrm{Cl}_{2}(4 \mathrm{~mL})$ under an argon atmosphere Dess-Martin periodinane $(59 \mathrm{mg}, 0.14 \mathrm{mmol}$ ) was added. The mixture was stirred at room temperature for $2 \mathrm{~h}$. The reaction was quenched with sat. $\mathrm{NaHCO}_{3}$ and $\mathrm{NaS}_{2} \mathrm{O}_{3} \cdot 5 \mathrm{H}_{2} \mathrm{O}$ solution $(10 \mathrm{~mL})$ and extracted with $\mathrm{CH}_{2} \mathrm{Cl}_{2}(2 \times 10 \mathrm{~mL})$. The combined extracts were dried $\left(\mathrm{Na}_{2} \mathrm{SO}_{4}\right)$, filtered and concentrated in vacuo to give dialdehyde $\mathbf{1 4}(14 \mathrm{mg}, 93 \%)$ as a yelloe oil; IR $\left(\mathrm{CH}_{2} \mathrm{Cl}_{2}\right) v_{\max }: 2962,1704,1463,1260 \mathrm{~cm}^{-1} ;{ }^{1} \mathrm{H}$ NMR $\delta 7.33(\mathrm{~d}, J=7.2 \mathrm{~Hz}, 1 \mathrm{H}), 7.28$ $(\mathrm{d}, J=7.2 \mathrm{~Hz}, 1 \mathrm{H}), 7.26(\mathrm{~s}, 1 \mathrm{H}), 7.20-7.08(\mathrm{~m}, 7 \mathrm{H}), 4.17(\mathrm{~s}, 1 \mathrm{H}), 3.60(\mathrm{dd}, J=9.8,5.6 \mathrm{~Hz}$, $1 \mathrm{H}), 3.24(\mathrm{dd}, J=10.8,9.8 \mathrm{~Hz}, 1 \mathrm{H}), 2.55(\mathrm{dd}, J=10.8,5.6 \mathrm{~Hz}, 1 \mathrm{H}), 0.69(\mathrm{~s}, 9 \mathrm{H}),-0.13$ (s, $3 \mathrm{H}),-0.17$ (s, 3H). ${ }^{13} \mathrm{C}$ NMR $\delta 204.4,201.2,148.8,145.4,144.6,141.4,140.1,138.9,126.8$, 126.6, 126.1, 126.0, 125.7, 125.5, 124.8, 123.0, 63.3, 55.2, 51.5, 51.1, 46.5, 25.8, -5.5, -5.6. HRCI-MS $m / z$ cald for $[\mathrm{M}+\mathrm{H}]^{+} \mathrm{C}_{27} \mathrm{H}_{31} \mathrm{O}_{3} \mathrm{Si}$ : 431.2042, found: 431.2035 .

\section{Results and Discussion}

Synthesis of cyclopentenone adduct began from Diels-Alder reaction between the know dimethyl fumarate and anthracene in xylene at $120^{\circ} \mathrm{C}$ for $48 \mathrm{~h}$ in a sealed tube gave ester 3 in $78 \%$ yield (Scheme 1). Reduction of the adduct 3 using $\mathrm{LiAlH}_{4}$ led to the alcohol 4 in quantitative yield. The di-alcohol $\mathbf{4}$ was protected as its monoacetate adduct $\mathbf{5}$ and transformed another alcohol group into carboxylic acid $\mathbf{6}$ by using Jone reagent as high as $91 \%$ yield (Scheme 2). Esterification of the carboxylic acid $\mathbf{6}$ with methanol in the present of 
$\mathrm{H}_{2} \mathrm{SO}_{4}$ into their methyl ester was however, obtained the alcohol 7 as the acetate protecting group was cleaved during this reaction. Thus, re-protected the $-\mathrm{OH}$ group of the resulting methyl ester $\mathbf{7}$ with $t$-butyldimethylsilyl chloride gave silyl adduct 8 in $81 \%$.

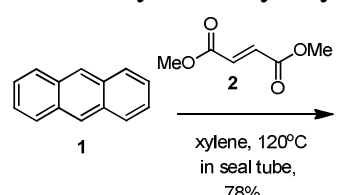

$78 \%$

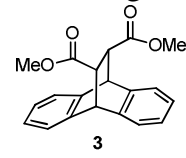

3 $\mathrm{Ac}_{2} \mathrm{O}$, pyridine,
$\mathrm{DMF}, 52 \%$

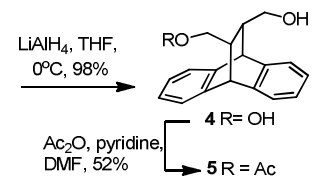
$\mathrm{CrO}_{3}$ in $\mathrm{H}_{2} \mathrm{O}$
$\mathrm{H}_{2} \mathrm{SO}_{4}, 91 \%$

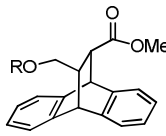

TBDMSCI, imidazole $\mathrm{CH}_{2} \mathrm{Cl}_{2}, 81 \%$

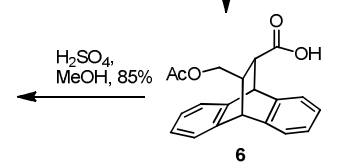

- $7 \mathrm{R}=\mathrm{H}$

$8 \mathrm{R}=\mathrm{TBDMS}$

Scheme 1. Synthetic route to methylester adduct 8

Spirocyclopenten-dione $\mathbf{1 4}$ required for this synthesis was prepared in a six-steps synthetic route starting with treatment of ester 8 with LDA in THF at $-78{ }^{\circ} \mathrm{C}$ in the presence of methyl cyanoformate to form dimthylester adduct 9 in $81 \%$ yield. This was reacted with $\mathrm{LiAlH}_{4}$ in THF at $0{ }^{\circ} \mathrm{C}$ forming the primary alcohol $\mathbf{1 0}(61 \%)$, which was further oxidized to the corresponding dialdehyde 11 by Dess-Martin periodinane, both steps proceeding in excellent yield. Compound $\mathbf{1 1}$ was then obtained by addition vinylmagnesium bromide to give secondary alcohol $\mathbf{1 2}(8.9 \%)$ as a mixture of four diastereomer, followed by olefin cross metathesis using Grubb's catalyst to give alkene 13 (53\%). Oxidation of di-alcohol 13 with Dess-Martin periodinane gave spirocyclopenten-dione 14 in 93\% yield (Scheme 2).

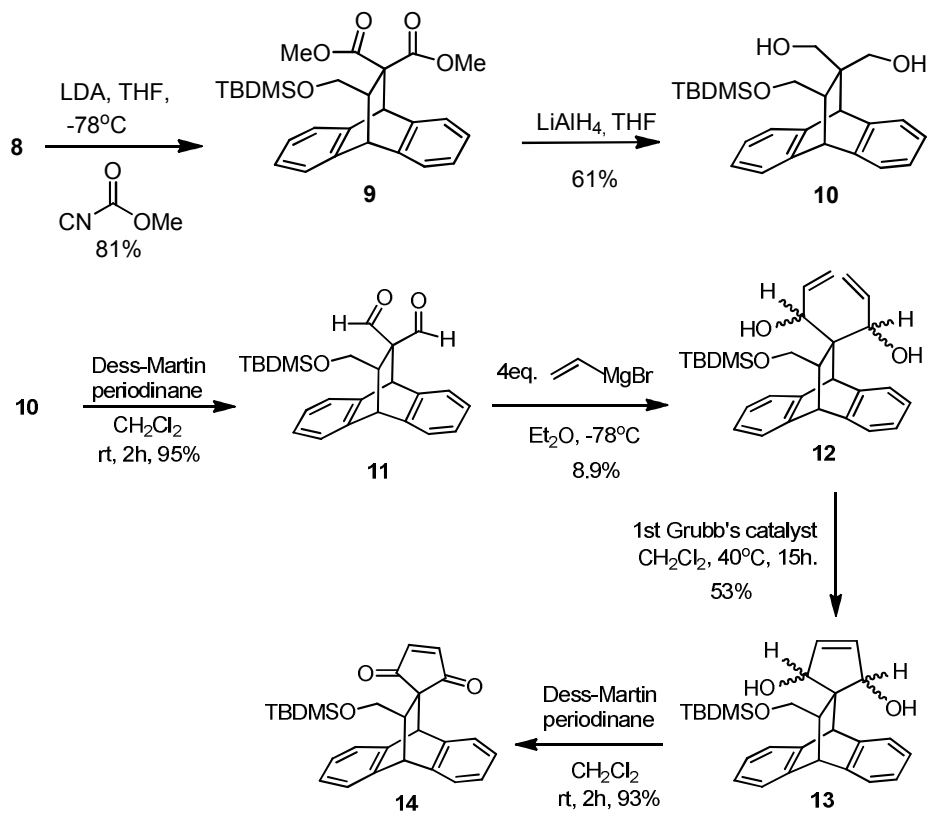

Scheme 2. Synthetic route to Spirocyclopenten-dione 14 


\section{Conclusion}

The spirocyclopenten-dione anthracene adduct (14), a precursor of the cyclopentenone prostaglandins, has been synthesized in 12 steps. This compound may in the future as a building block to provide an efficient route for the synthesis of prostanoid natural products.

\section{Acknowledgement}

We gratefully acknowledge support from The Thailand Research Fund (Research grant No. RMU5080006), Silpakorn University and the University of Wollongong and the Department of Chemistry, Faculty of Science, Silpakorn University for a scholarship (to K.E.).

\section{References}

1. Kikuchi H, Tsukitani Y, Iguchi K and Yamada Y, Tetrahedron Lett., 1982, 23(49), 5171-5174.

2. Kikuchi H, Tsukitani Y, Iguchi K and Yamada Y, Tetrahedron Lett., 1983, 24(14), 1549-1552.

3. (a) Blumenkopf $\mathrm{T}$ A and Overman L E, Chem Rev., 1986, 86(5), 857-873;

(b) Habermas K L, Denmark S E and Jones T K, Org React., 1994, 45, 1.

4. Lin Y S, Khalil A T, Chiou S H, Kuo Y C, Cheng Y B, Liaw C C and Shen Y C, Chemistry Biodiversity, 2008, 5, 784-792.

5. $\quad$ Nakajima A, Takeda K and Yoshii E, Synlett., 1997, 255.

6. Davie C P and Danheiser R L, Angew Chem Int Ed Engl., 2005, 44(36), 5867-5870.

7. Thebtaranonth Y, Pure Appl Chem., 1997, 69, 609-614.

8. Phutdhawong W, Pyne S G, Baramee A, Buddhasukh D and Willis A C, Tetrahedron Lett., 2002, 43(34), 6047-6049.

9. Shen Y C, Cheng Y B, Lin Y C, Guh J H, Teng C M and Ko C L, J Nat Prod., 2004, 67(4), 542-546.

10. Kimpe N D, Palamareva M and Schamp N, J Org Chem., 1985, 50(16), 2993-2995. 